\title{
Theory of Ionization By Cumulative Action.
}

\author{
By K. T. Compton.
}

A STUDY of the ionization in a gas subjected to thermionic bombardment between an incandescent cathode and an anode enables certain features of the process of the ionization to be determined. In case of a metallic vapor at more than a couple of millimeters pressure and at moderate voltages, it is readily shown that but a negligible part of the ionization can be due to single direct electronic impacts against atoms. Practically all the ionization is due to ionization of atoms which have previously been partially ionized in the process of producing resonance radiation by a preceding impact or by absorption of such radiation produced by impacts against neighboring molecules.

Under such conditions, and if the thermionic emission is limited by space charge, it is shown that the total number of emitted electrons is:

$$
n=\frac{n_{0}}{\mathrm{I}-4 \sqrt{2} \sqrt{\mathrm{I} 840 M} \pi P_{0}}
$$

where $M$ is the molecular weight of the gas (on the basis $M_{H}=\mathrm{I}$ ), $n_{0}$ is the number of electrons which would be emitted under otherwise similar conditions if there were no ionization, $P_{0}$ is the probability that any molecule may be in the abnormal or partially ionized condition, and $\pi$ is a constant which is probably unity for a pure monatomic gas or vapor. By this relation, $P_{0}$ may be experimentally determined.

If $n_{0}$ be increased, $P_{0}$, which increases rapidly with $n_{0}$, increases until the thermionic current $n$ would become infinite if it were not limited by the saturation emission at the given temperature. Owing to such limitation, it is shown that the space charge changes sign, with a further increase in current. The maximum arc current cannot exceed two times the saturation value of thermionic emission and, at voltages less than the normal ionizing potential, is probably about one and a half times the thermionic saturation current.

Expressions for $P_{0}$ are derived on the assumptions (I) that the abnormal state is the result of a preceding impact against the same molecule and (2) that it results from absorption of resonance radiation. When applied to the particular case of a cathode passing axially inside a cylindrical anode, calculations show that process (2) is much more important than process (I).

This ionization by cumulative action, in which the effect of radiation is very important, is probably of preponderating importance in temperature ionization of gases.

A full report of this work is soon to be published.

PRINCETON UNIVERSITY. 